\title{
Grazing incidence wavefront sensing and verification of X-ray optics performance
}

\author{
Timo T. Saha, Scott Rohrbach, and William W. Zhang, NASA Goddard Space Flight \\ Center, Greenbelt, Md 20771
}

Evaluation of interferometrically measured mirror metrology data and characterization of a telescope wavefront can be powerful tools in understanding of image characteristics of an x-ray optical system. In the development of soft X-ray telescope for the International X-Ray Observatory (IXO), we have developed new approaches to support the telescope development process.

Interferometrically measuring the optical components over all relevant spatial frequencies can be used to evaluate and predict the performance of an x-ray telescope. Typically, the mirrors are measured using a mount that minimizes the mount and gravity induced errors. In the assembly and mounting process the shape of the mirror segments can dramatically change. We have developed wavefront sensing techniques suitable for the $\mathrm{x}$-ray optical components to aid us in the characterization and evaluation of these changes.

Hartmann sensing of a telescope and its components is a simple method that can be used to evaluate low order mirror surface errors and alignment errors. Phase retrieval techniques can also be used to assess and estimate the low order axial errors of the primary and secondary mirror segments.

In this paper we describe the mathematical foundation of our Hartmann and phase retrieval sensing techniques. We show how these techniques can be used in the evaluation and performance prediction process of $\mathrm{x}$-ray telescopes. 


\section{Grazing incidence wavefront}

sensing and verification of $x$-ray

\section{optics performance}

Timo T. Sahaa, Scott Rohrbacha, William W. Zhanga ${ }^{a}$ Tyler C. Evans ${ }^{b}$, and Melinda Hong ${ }^{b}$ aNASA/Goddard Space Flight Center, 8800 Greenbelt Road, Greenbelt, MD 20771 bSGT, Inc, 7701 Greenbelt Road Suite 400, Greenbelt MD 20 


\section{Outline}

- Objective is to develop complete set of analysis tools for grazing incidence optics including alignment/assembly process and system optical performance verification and modeling

- Wavefront sensing technique based on Hartmann tests of x-ray mirrors of the International X-ray Observatory (IXO)

- Alignment errors considered

- Description of the analysis tool

- Modeling example

- Performance modeling principles and analysis developed for segmented IXO x-ray mirrors

- Basic equations

- Performance analysis of a mirror pair

- Phase retrieval analysis of segmented IXO X-ray mirrors

- Description of the phase retrieval process

- Preliminary results 
Alignment errors and Hartmann test 


\section{Defocus error}

- Displacement of the focal plane in axial direction

- Approximate ray intercept equations

- $H_{x}=\Delta_{2} \tan \left(\alpha_{0}\right) \sin (\beta)$

- $H_{y}=\Delta_{z} \tan \left(\alpha_{0}\right) \cos (\beta)$.

- For 360-degree optic the centroid map is just a circle in the focal plane

- For 30-degree segmented optic the centroid map is 30-degree arc in the focal plane

- Positive and negative defocus is shown in the focal plane plot
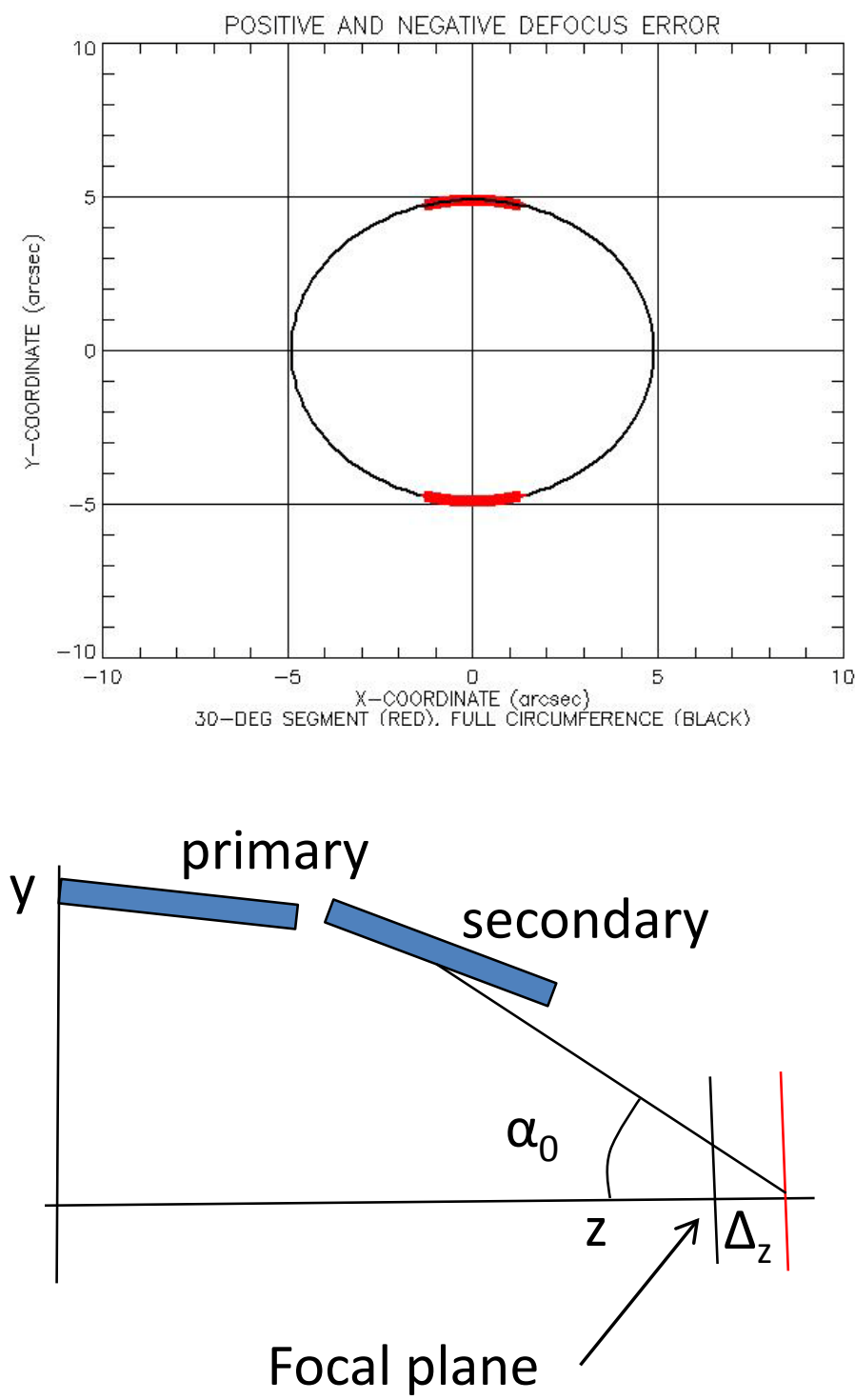


\section{X-Decenter error}

- Secondary moves along $\mathrm{x}$-axis

- Approximate equations

$-H_{x}=2 \Delta_{x}-\Delta_{x} \cos (2 \beta)$

- $H_{y}=\Delta_{x} \sin (2 \beta)$

- For full circumference optic x-decenter generates double circle at the focal plane. The double circle is offset twice the radius of the circle along $\mathrm{x}$-axis

- 30-degree segments generate 60-degree arcs

- Center of the arc is a radius away from the optical axis

- Images of positive and negative errors are shown

- Image footprint is long in vertical direction

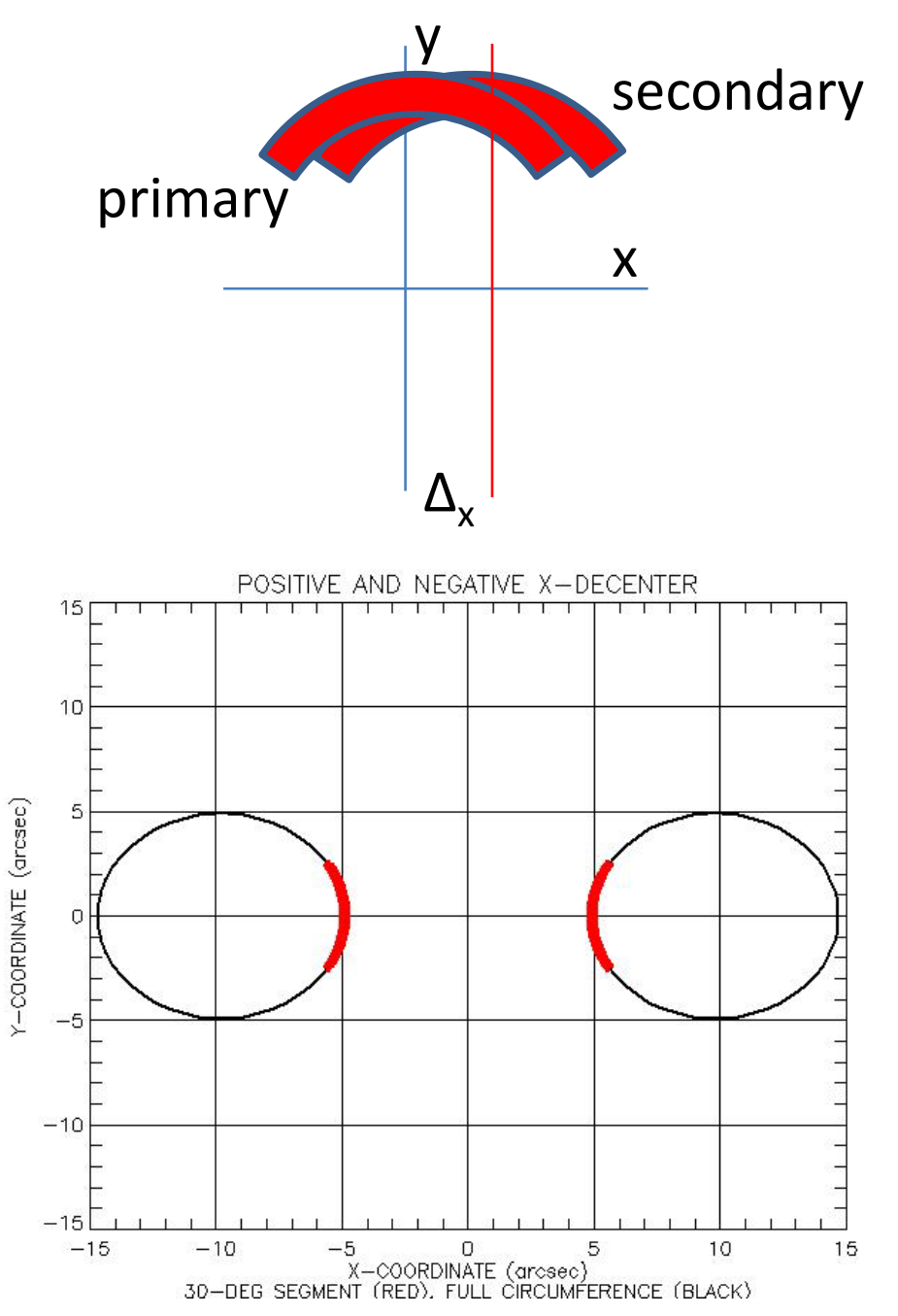




\section{Y-Decenter error}

- Secondary is displaced along y-axis

- Approximate ray intercepts at focal plane

- $H_{x}=\Delta_{y} \sin (2 \beta)$

$-H_{y}=2 \Delta_{y}+\Delta_{y} \cos (2 \beta)$

- 360-degree optic generates double circle in the focal plane. The circles are displaced along $y$-axis by a distance that is twice the radius of the circle

- 30-degree segment generates 60-degree arc in the focal plane. The arc is located 3 radii away along $y$-axis from the optical axis

- Images of positive and negative errors are shown

- Image footprint is long in horizontal direction
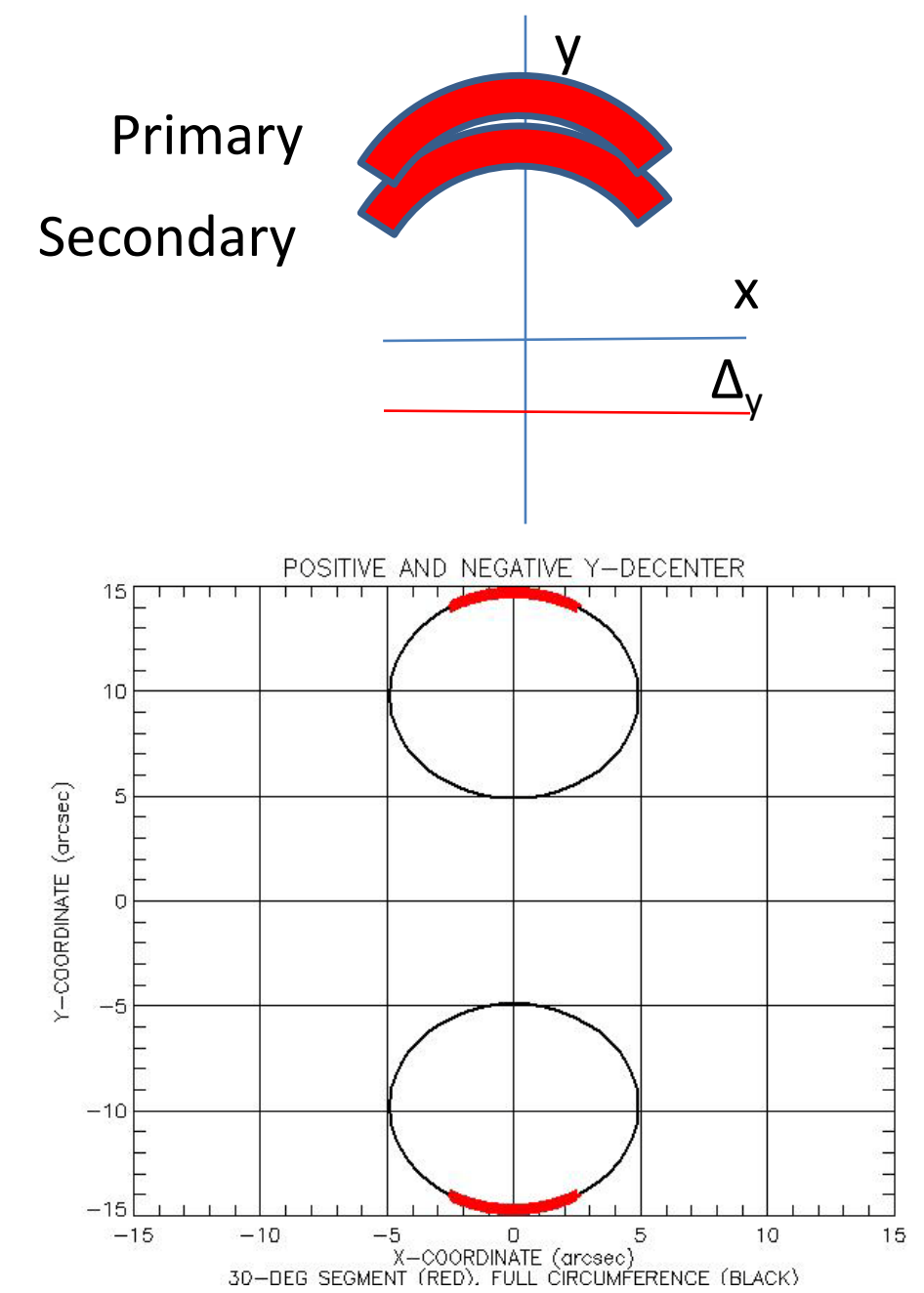


\section{Azimuth alignment error}

- Secondary rotates about y-axis in x-z-plane

- Approximate equations

- $H_{x}=f \delta_{x}-f \delta_{x} \cos (2 \beta)$

- $H_{y}=f \delta_{x} \sin (2 \beta)$

- 360-degree optic generates double circle image. The circle is offset along $x$-axis by a distance that is the radius of the circle

- Image of the 30-degree segmented optic is a secondary 60 -degree arc. The center of the arc is on the optical axis

- Images of positive and negative errors are shown

- Image footprint is long in vertical direction

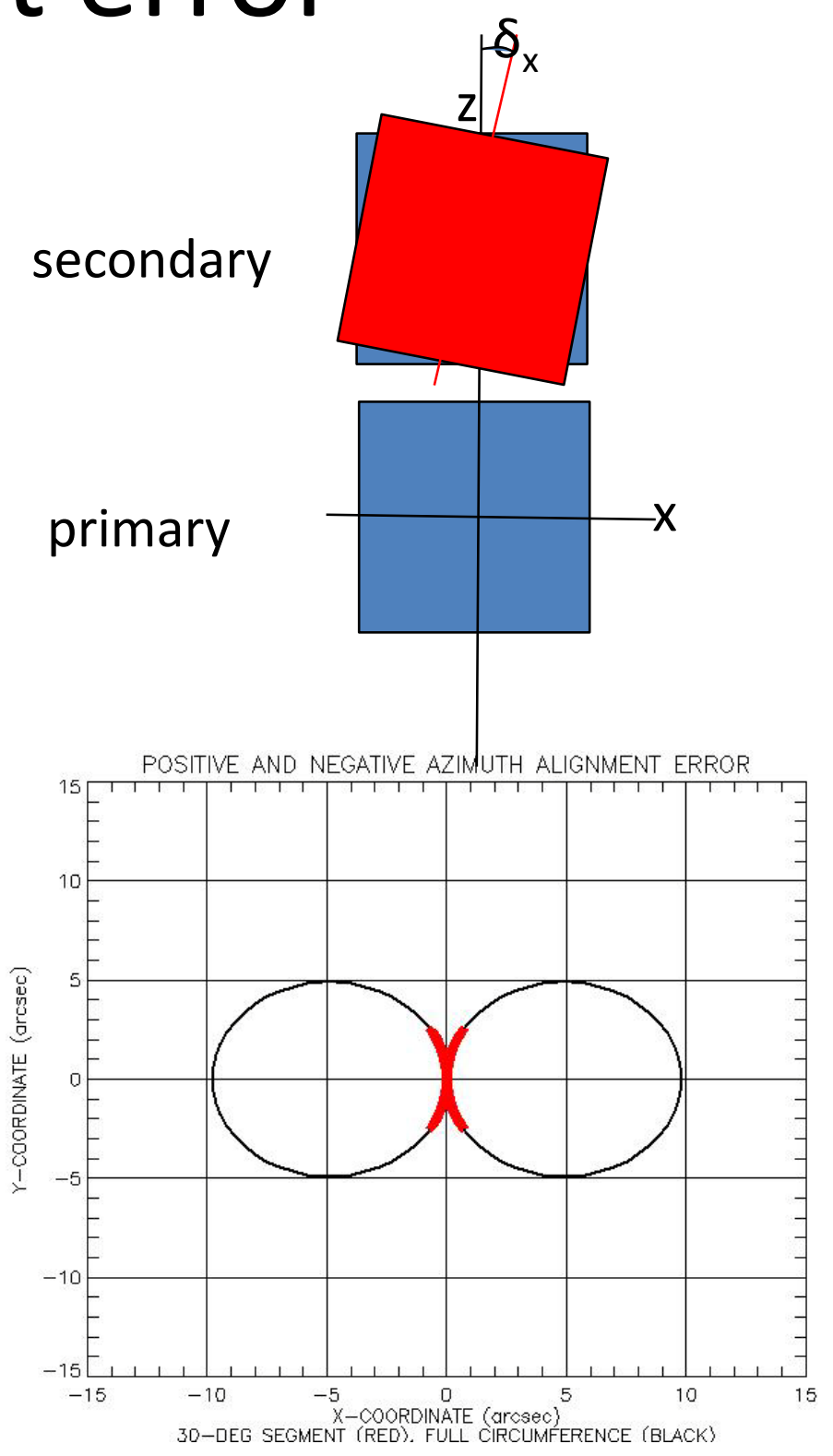




\section{Elevation alignment error}

- Secondary rotates about $x$-axis in y-z-plane

- Equations

- $H_{x}=f \delta_{y} \sin (2 \beta)$

- $H_{y}=f \delta_{y}+f \delta_{y} \cos (2 \beta)$

- Image generated by 360-degree optic is a double circle centered a radius away from optical axis along the y-direction

- Images of positive and negative errors are shown

- Image generated by 30-degree segmented optic is a 60-degree arc located 2 radii away from the optical axis

- Image footprint is long in horizontal direction
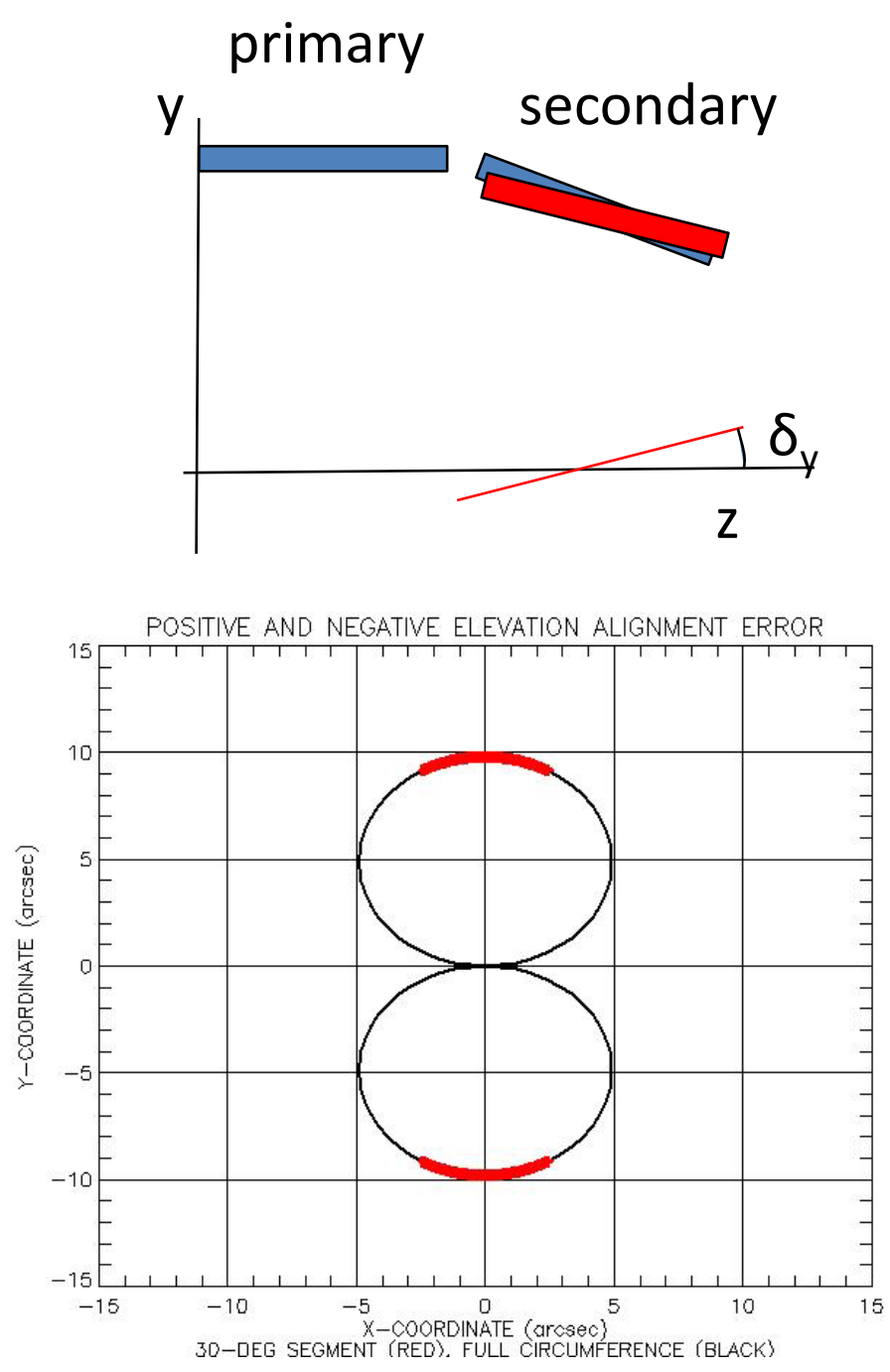


\section{Retrieval of alignment errors}

- Assuming alignment errors are small the ray interception coordinates $\left(\mathrm{H}_{\mathrm{x}}, \mathrm{H}_{\mathrm{y}}\right)$ are simply added

- $H_{x}, H_{y}$ and $\beta$ are determined from the Hartmann scan data

- $\mathrm{H}_{\text {plus }}$ and $\mathrm{H}_{\text {minus }}$ are fit into trigonometric functions and the alignment parameters are found

$$
\begin{aligned}
& H_{x}=2 \Delta_{x}+\Delta_{z} \tan \left(\alpha_{0}\right) \sin (\beta)+f \delta_{x}+\left(\Delta_{y}+f \delta_{y}\right) \sin (2 \beta)-\left(\Delta_{x}+f \delta_{x}\right) \cos (2 \beta) \\
& H_{y}=2 \Delta_{y}+\Delta_{z} \tan \left(\alpha_{0}\right) \cos (\beta)+f \delta_{y}+\left(\Delta_{x}+f \delta_{x}\right) \sin (2 \beta)+\left(\Delta_{y}+f \delta_{y}\right) \cos (2 \beta) \\
& H_{\text {plus }}=H_{x} \sin (\beta)+H_{y} \cos (\beta) \\
& H_{\text {minus }}=H_{x} \cos (\beta)-H_{y} \sin (\beta) \\
& H_{\text {plus }}=\Delta_{z} \tan \left(\alpha_{0}\right)+\left(3 \Delta_{x}+2 f \delta_{x}\right) \sin (\beta)+\left(3 \Delta_{y}+2 f \delta_{y}\right) \cos (\beta) \\
& H_{\text {minus }}=\Delta_{x} \cos (\beta)-\Delta_{y} \sin (\beta)
\end{aligned}
$$




\section{Testing of retrieval process}

- Used OSAC ray trace software in the testing of the retrieval process

- Calculated the image centroids $\left(\mathrm{H}_{\mathrm{x}}, \mathrm{H}_{\mathrm{y}}\right)$, variables $\mathrm{H}_{\text {plus }}$ and $\mathrm{H}_{\text {minus }}$, and azimuth angle $\beta$ from the OSAC output

- Fit the data to the equations

- Alignment errors and image centroid coordinates are retrieved within 1$2 \%$

\begin{tabular}{|l|l|l|}
\hline & Introduced error & Retrieved error \\
\hline Defocus (mm) & 30.00 & 30.11 \\
\hline Elevation alignment (arc-sec) & 10.00 & 9.89 \\
\hline Azimuth alignment (arc-sec) & 5.00 & 4.94 \\
\hline X-decenter $(\boldsymbol{\mu m})$ & 20.00 & 19.99 \\
\hline Y-decenter $(\boldsymbol{\mu m})$ & 10.00 & 9.85 \\
\hline Image x-centroid $(\mathrm{mm})$ & 0.221 & 0.221 \\
\hline Image y-centroid $(\mathrm{mm})$ & 0.413 & 0.413 \\
\hline
\end{tabular}


Optical performance modeling 


\section{Principles of performance modeling}

- Determine grazing angles $i_{p}$ of the primary and $i_{s}$ of the secondary

- Calculate the OPD from measured

$$
I(\xi)=\int_{-\infty}^{\infty} e^{i \frac{2 \pi}{\lambda} O P D(r)} e^{-i \frac{2 \pi}{R \lambda} \xi r} d r
$$
surface errors $D_{p}$ and $D_{s}$

- Radial intensity profiles are then calculated from the diffraction integral

$$
O P D=2 D_{p} \sin \left(i_{p}\right)+2 D_{s} \sin \left(i_{s}\right)
$$

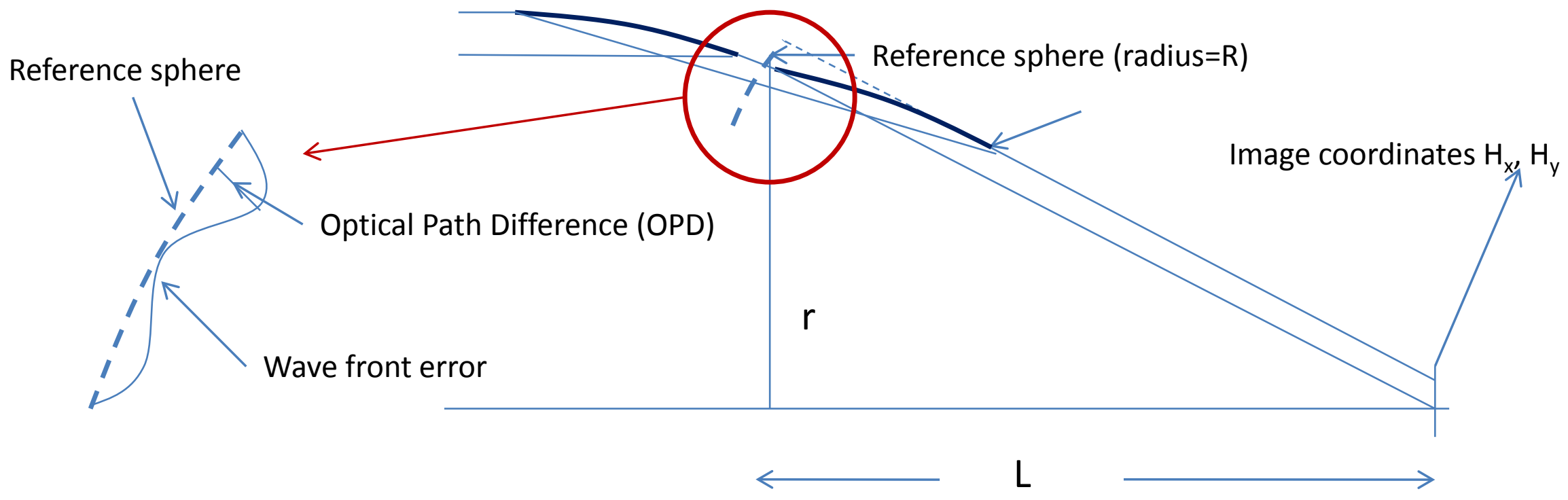




\section{Mirror surface errors}

- Dimensions of the mirrors are $200 \mathrm{~mm}$ by 30 degrees

- Surface figure errors of mirror segments

- Measured interferometrically using corrective cylindrical null lens

- Used 4-point mount and the mirrors are oriented optical axis vertical

- Axial sag error is dominating feature in the primary mirror segment

- High zone replicated from the mandrel is visible in the secondary mirror segment in 20 $-50 \mathrm{~mm}$ axial range
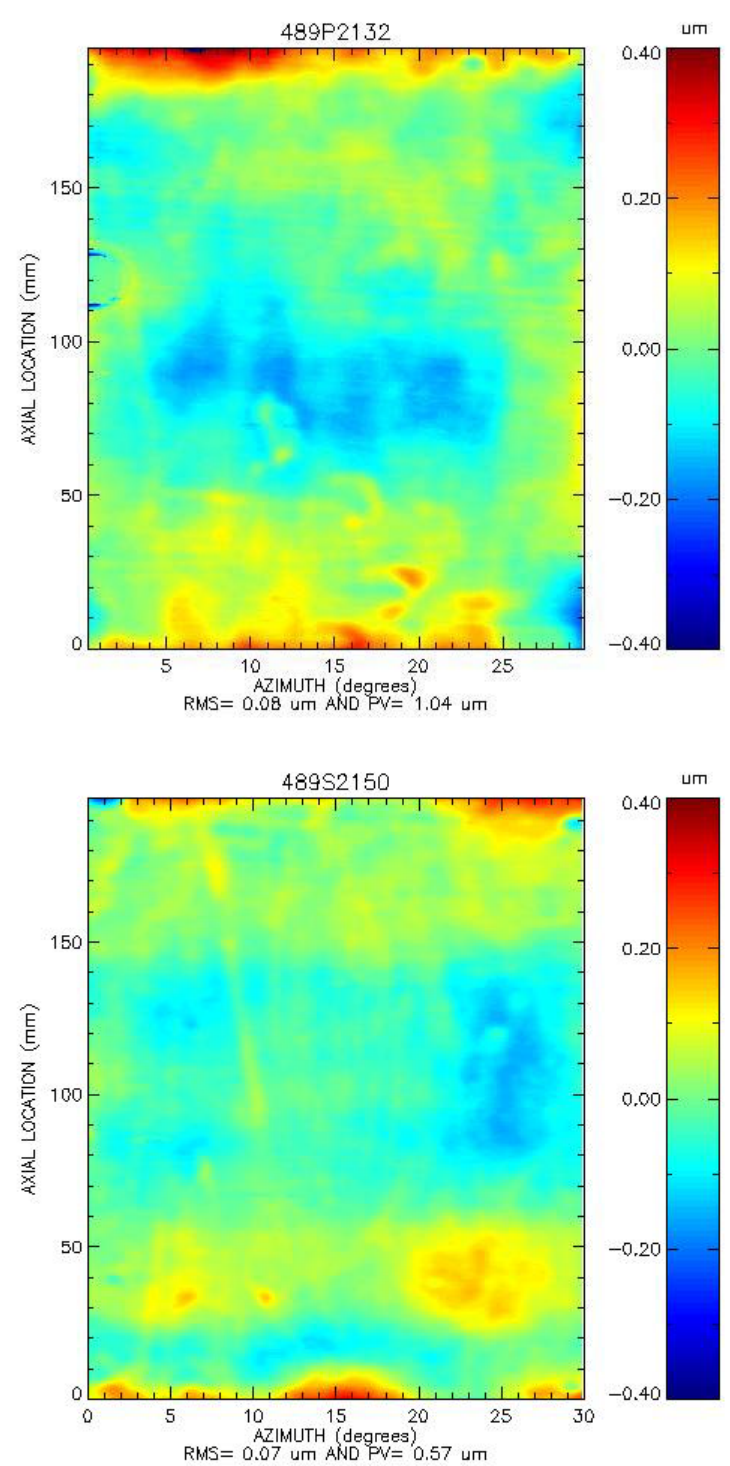


\section{Image and half power diameter (HPD)}

- Optical performance modeled at $1.0 \mathrm{KeV}$

- Image is narrow 30-degree bowtie

- HPD of this mirror pair is 5.1 arc-sec

- Secondary mirror segment contribute slightly less to the HPD of the telescope
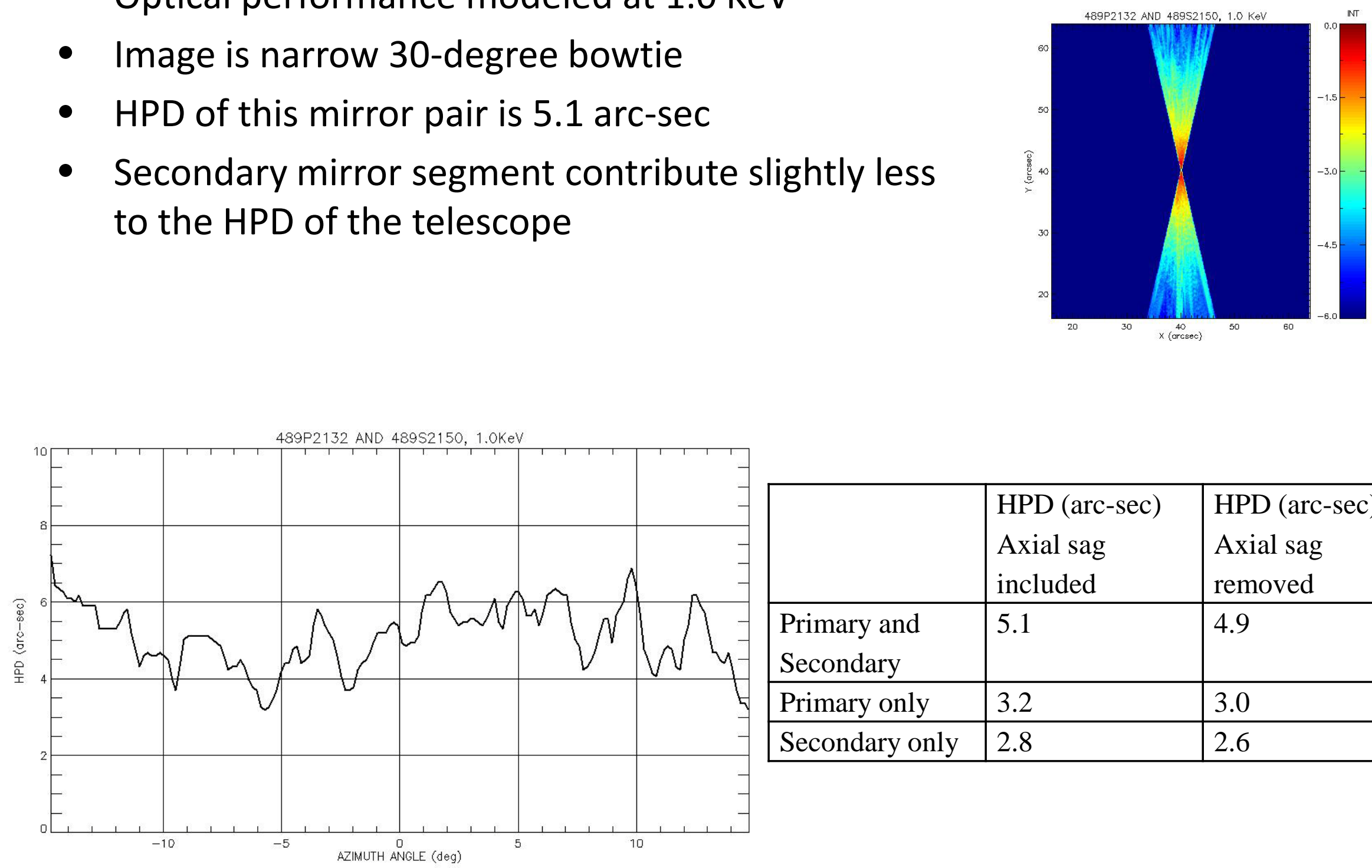

\begin{tabular}{|l|l|l|}
\hline & $\begin{array}{l}\text { HPD (arc-sec) } \\
\text { Axial sag } \\
\text { included }\end{array}$ & $\begin{array}{l}\text { HPD (arc-sec) } \\
\text { Axial sag } \\
\text { removed }\end{array}$ \\
\hline $\begin{array}{l}\text { Primary and } \\
\text { Secondary }\end{array}$ & 5.1 & 4.9 \\
\hline Primary only & 3.2 & 3.0 \\
\hline Secondary only & 2.8 & 2.6 \\
\hline
\end{tabular}


Phase retrieval 


\section{Principles of phase retrieval}

- Using non-linear curve fitting techniques OPD is estimated from the measured radial intensity profile

- The axial surface error $D_{p}$ is then estimated from the OPD

$$
\begin{aligned}
& I(\xi)=\int_{-\infty}^{\infty} e^{i \frac{2 \pi}{\lambda} O P D(r)} e^{-i \frac{2 \pi}{R \lambda} \xi r} d r \\
& O P D=2 D_{p} \sin \left(i_{p}\right)
\end{aligned}
$$




\section{Measured Hartmann image}

- Plot shows a Hartmann image of the secondary mirror measured at $633 \mathrm{~nm}$

- Image is long in radial direction because the secondary segment does not focus the collimated beam

- Diffraction coming from the radial height of the segment is visible in the radial direction

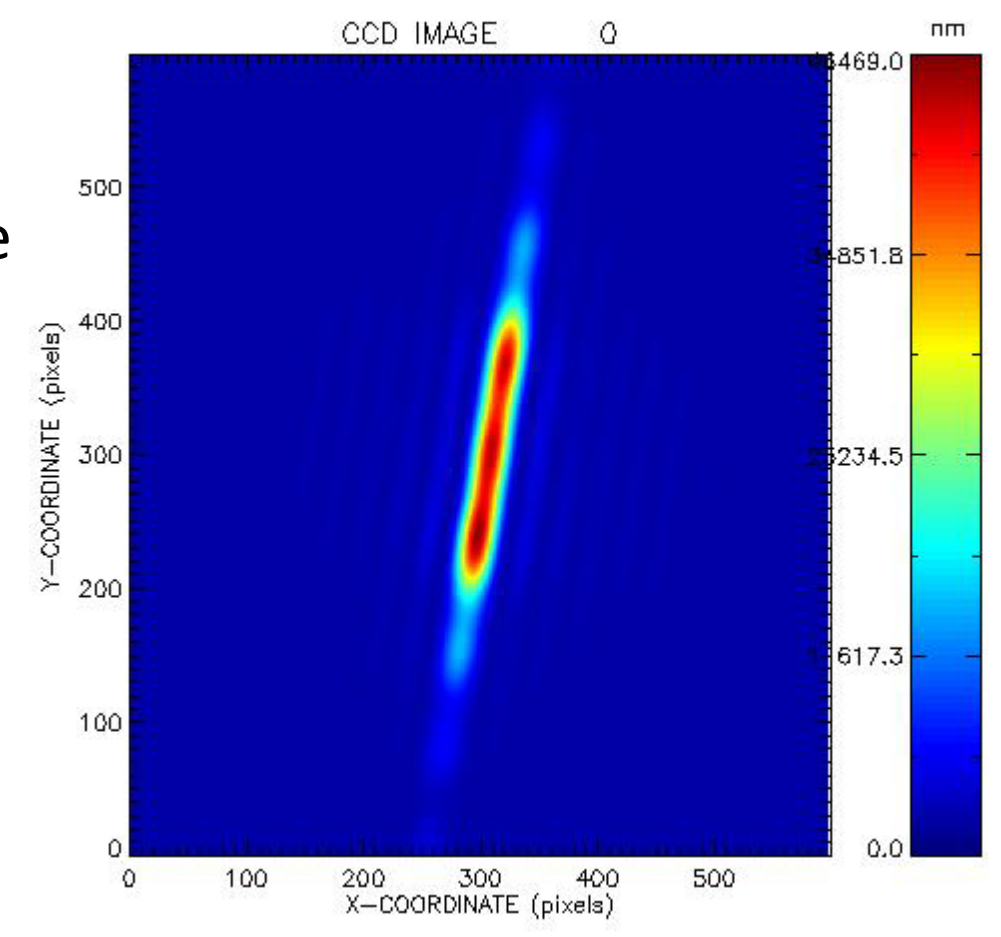

- Diffraction coming from the width of the slit is visible in the tangential direction

- Extracted a single profile along radial direction. This profile is used as input data for the phase retrieval algorithm 


\section{Preliminary results}

- Surface plot on the left shows the figure error of the mirror measured interferometrically

- Radial intensity profiles were measured in 11 locations along the azimuth

- Surface plot on the right shows the surface error retrieved from the intensity profiles

- Low order axial errors in $0.1 \mu \mathrm{m}$ range can be retrieved from the radial intensity profiles
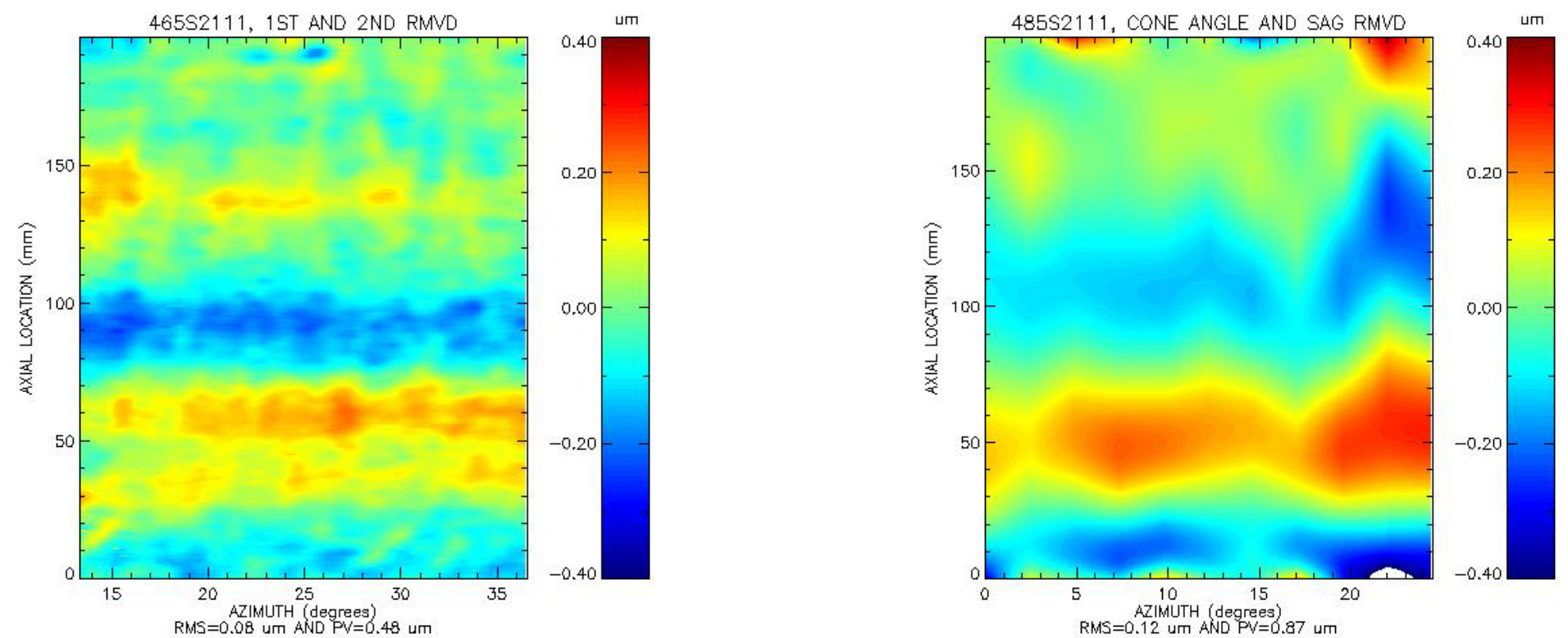


\section{Conclusions}

- Analysis tool developed for the Hartmann scan data

- Accurately retrieves the alignment errors

- Facilitates the telescope assembly and alignment process and the tool has been integrated into the IXO mirror development process

- Optical performance modeling software

- Tool is routinely used in the development of IXO mirrors to evaluate the effects of low and high frequency errors on the telescope performance

- Phase retrieval software

- Developed technique can be used to evaluate low order axial surface errors

- Phase retrieval can be very useful technique for the nested $x$-ray telescopes since no normal incidence access to the mirrors is required 